\title{
DECAY OF A $p$-HARMONIC MEASURE IN THE PLANE
}

\author{
Niklas L. P. Lundström and Jonatan Vasilis \\ Umeå University, Department of Mathematics and Mathematical Statistics \\ SE-901 87 Umeå, Sweden; niklas.lundstrom@math.umu.se \\ Chalmers University of Technology, Department of Mathematical Sciences \\ and University of Gothenburg, Department of Mathematical Sciences \\ SE-412 96 Göteborg, Sweden
}

\begin{abstract}
We study the asymptotic behaviour of a $p$-harmonic measure $\omega_{p}, p \in(1, \infty]$, in a domain $\Omega \subseteq \mathbf{R}^{2}$, subject to certain regularity constraints. Our main result is that $\omega_{p}(B(w, \delta) \cap$ $\left.\partial \Omega, w_{0}\right) \approx \delta^{q}$ as $\delta \rightarrow 0^{+}$, where $q=q(v, p)$ is given explicitly as a function of $v$ and $p$. Here, $v$ is related to properties of $\Omega$ near $w$. If $p=\infty$, this extends to some domains in $\mathbf{R}^{n}$. By a result due to Hirata, our result implies that the $p$-Green function for $p \in(1,2)$ is not quasi-symmetric in plane $C^{1,1}$-domains.
\end{abstract}

\section{Introduction}

Let $\Omega \subset \mathbf{R}^{n}$ be a regular bounded domain and let $f$ be a real-valued continuous function defined on $\partial \Omega$. It is well known that there exists a unique smooth function $u$, harmonic in $\Omega$, such that $u=f$ continuously on $\partial \Omega$. The maximum principle and the Riesz representation theorem yield the following representation formula for $u$,

$$
u(z)=\int_{\partial \Omega} f(w) \mathrm{d} \omega^{z}(w), \quad \text { whenever } z \in \Omega .
$$

Here, $\omega^{z}(w)=\omega(d w, z, \Omega)$ is referred to as the harmonic measure at $z$ associated to the Laplace operator. As the harmonic measure allows us to solve the Dirichlet problem, its properties are of fundamental interest in classical potential theory.

Consider the harmonic measure at some fixed point $z$, a natural question is: How does the harmonic measure of a $\delta$-neighborhood around a given boundary point decay when $\delta$ tends to zero? Or, expressed in probabilistic terms, what is the probability that a Brownian motion started at $z$ will first hit the boundary in a $\delta$-neighborhood around the boundary point? For smooth domains, a classical result is that the probability is comparable to $\delta^{n-1}$, when $\delta$ is small.

In this paper we study decay of a $p$-harmonic measure, defined below, which is a generalization of harmonic measure, related to the $p$-Laplace equation. For $p \in(1, \infty)$, the $p$-Laplace equation yields

$$
\Delta_{p} u:=\nabla \cdot\left(|\nabla u|^{p-2} \nabla u\right)=0
$$

If $p=\infty$, then the equation is no longer of divergence form and can be written as

$$
\Delta_{\infty} u:=\sum_{i, j=1}^{n} \frac{\partial u}{\partial x_{i}} \frac{\partial u}{\partial x_{j}} \frac{\partial^{2} u}{\partial x_{i} \partial x_{j}}=0,
$$

doi:10.5186/aasfm.2013.3808

2010 Mathematics Subject Classification: Primary 35J25, 35J70.

Key words: Harmonic measure, harmonic function, $p$-Laplace operator, generalized interior ball. 
which is the so called $\infty$-Laplacian. For the definition of solutions to the $p$-Laplacian, that is, weak solutions, viscosity solutions and $p$-harmonicity, we refer the reader to Section 3.

Definition 1.1. Let $\Omega \subseteq \mathbf{R}^{n}$ be a domain, $E \subseteq \partial \Omega, p \in(1, \infty)$, and $w_{0} \in \Omega$. The $p$-harmonic measure of $E$ at $w_{0}$ with respect to $\Omega$, denoted $\omega_{p}\left(E, w_{0}, \Omega\right)$, is defined as $\inf _{u} u\left(w_{0}\right)$, where the infimum is taken over all $p$-superharmonic functions $u \geq 0$ in $\Omega$ such that $\liminf _{z \rightarrow w} u(z) \geq 1$, for all $w \in E$.

The $\infty$-harmonic measure is defined in a similar manner, but with $p$-superharmonicity replaced by absolutely minimizing (AM), see [PSSW09, pp. 173-174]. To avoid confusion, we mention that there are at least three different definitions of $p$ harmonic measure in the literature. Besides the $p$-harmonic measure above, we refer to the definitions given in [BL05] and [HK97]. In the rest of the paper, we write $p$-harmonic measure to mean $\omega_{p}\left(E, w_{0}, \Omega\right)$ as defined in Definition 1.1, and if the underlying domain is clear from context, we allow ourselves to write $\omega_{p}\left(E, w_{0}\right)$ in place of $\omega_{p}\left(E, w_{0}, \Omega\right)$. It turns out that $\omega_{p}(E, \cdot)$ is $p$-harmonic in $\Omega$ and that $0 \leq$ $\omega_{p}\left(E, w_{0}\right) \leq 1$, for all $w_{0} \in \Omega$. For these and other properties of $p$-harmonic measure, as well as for the fundamentals of the $p$-Laplace operator, we refer to [HKM93].

When $p \neq 2$, the $p$-harmonic measure fails to be a measure, and it no longer provides a solution formula for the Dirichlet problem, owing to the nonlinearity of the $p$-Laplace operator. Nevertheless, it can still be used to estimate solutions to the $p$-Laplace equation. For instance suppose that $u$ is $p$-subharmonic in $\Omega$, with $\limsup _{z \rightarrow w} u(z)$ bounded by $C$ when $w \in E \subseteq \partial \Omega$, and by $c \leq C$ on the rest of the boundary. Then $u \leq(C-c) \omega_{p}(E, \cdot)+c$ in $\Omega$, see [HKM93, Theorem 11.9].

The $p$-harmonic measure also has a probabilistic interpretation, this time in terms of the zero-sum two-player game tug-of-war [PS08, PSSW09]. Roughly speaking, given a domain in $\mathbf{R}^{n}, n \geq 2$, and a starting point, two players take turns in choosing an $\epsilon$-step, which is then perturbed by a $p$-dependent noise vector. The game ends when one of the players is able to reach the boundary of the domain. Player one receives a payoff of $\$ 1$ from player two if the game ends at a part $E$ of the boundary, otherwise neither player receives any payoff. As $\epsilon \rightarrow 0^{+}$, the value of this game tends to the $p$-harmonic measure of $E$.

Now consider a domain in $\mathbf{R}^{2}$. Our main result (Theorem 2.1) is that the $p$ harmonic measure of a $\delta$-neighborhood of a boundary point, satisfying certain regularity conditions, decays as a certain power of $\delta$, when $\delta \rightarrow 0^{+}$. For the upper bound, the condition on the domain is simply that it is contained in a sector with a certain aperture and with apex at the boundary point. The lower bound requires instead that the domain satisfies a generalized interior ball condition. Before giving the definition of the generalized interior ball, we introduce the sector $\mathcal{S}_{v}$, having aperture $\pi / v$ and apex at the origin. Let $\phi \in(-\pi, \pi]$ be the polar angle of $(x, y)$, then

$$
\mathcal{S}_{v}=\left\{(x, y) \in \mathbf{R}^{2} \backslash\{(0,0)\} ;|\phi|<\frac{\pi}{2 v}\right\} \quad \text { where } v \geq \frac{1}{2} .
$$

We now give the definition of the generalized interior ball condition. Here, as in the sequel, $(r, \phi)$ are polar coordinates for $(x, y)$.

Definition 1.2. Let $\Omega \subseteq \mathbf{R}^{2}$ be a domain and let, for $\gamma, r_{1}>0$ and $v \geq 1 / 2$,

$$
E\left(\gamma, r_{1}, v\right)=\left\{(x, y) \in \mathbf{R}^{2} ; \cos (v \phi)>\left(\frac{r}{r_{1}}\right)^{\gamma},|\phi|<\frac{\pi}{2 v}\right\} .
$$


A boundary point $w \in \partial \Omega$ is said to satisfy the generalized interior ball condition of type $\left(\gamma, r_{1}, v\right)$, if there exists a rigid transformation $T$ of the plane such that $T\left(E\left(\gamma, r_{1}, v\right)\right) \subseteq \Omega$ and $w=T(0,0)$. We then say that $w \in \partial \Omega$ is of type $\left(\gamma, r_{1}, v\right)$ for $\Omega$.

The set $E\left(\gamma, r_{1}, v\right)$ is by definition contained in the sector $\mathcal{S}_{v}$ defined in (1.2). On the boundary $\partial E\left(\gamma, r_{1}, v\right)$ we have that $r=r_{1} \cos ^{1 / \gamma}(v \phi)$, see Figure 1 . If $v=\gamma=1$, this is just a circle with radius $r_{1} / 2$ and center $\left(r_{1} / 2,0\right)$ and hence, in this case Definition 1.2 yields the usual interior ball condition. We also see that if $w \in \partial \Omega$ is of type $\left(\gamma, r_{1}, v\right)$, then it is also of type $\left(\gamma^{\prime}, r_{1}^{\prime}, v^{\prime}\right)$, for all $\gamma \geq \gamma^{\prime}>0, r_{1} \geq r_{1}^{\prime}>0$, and $1 / 2 \leq v \leq v^{\prime}$. Finally, it is not too difficult to verify that $E\left(\gamma, r_{1}, v\right)$ is convex if and only if $v \geq 1$, but that the parts of $E\left(\gamma, r_{1}, v\right)$ that are in the upper and lower half plane, respectively, are both convex for all $v \geq 1 / 2$. In particular, $E\left(\gamma, r_{1}, v\right)$ is star-shaped around $(x, 0)$, for all $x \in\left[0, r_{1}\right]$.

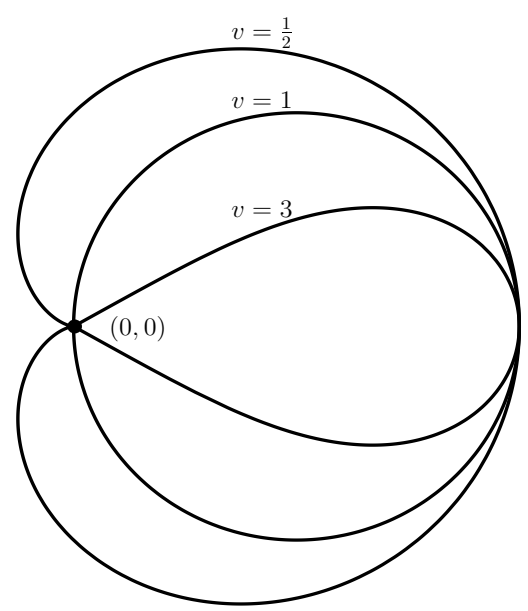

(a) $p=2$

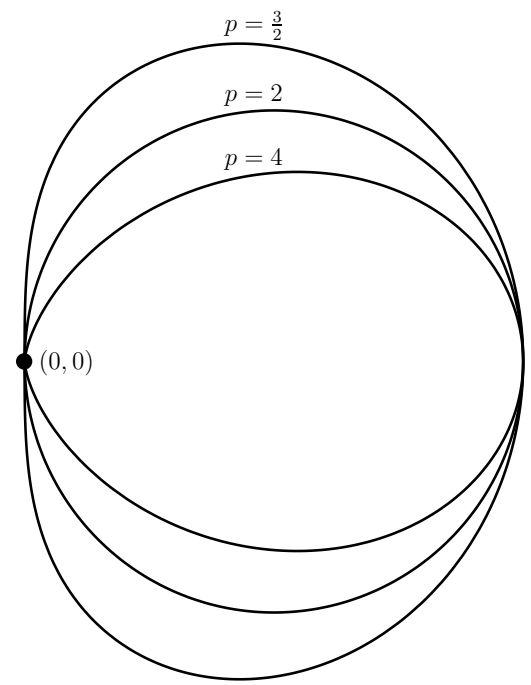

(b) $v=1$

Figure 1. The boundaries of the bounded sets $E\left(q, r_{1}, v\right)$, where $q=q(v, p)$ is as in (2.1), for various values of $p$ and $v$. The lower bound in Theorem 2.1 requires that $E\left(q, r_{1}, v\right)$ can be mapped by rotating and translating to a subset of $\Omega$, with $(0,0)$ mapped to a given boundary point of $\Omega$.

The rest of the paper is structured as follows. In Section 2 we present our main result (Theorem 2.1) and a number of Corollaries. In Section 3 we present some well known results and definitions for $p$-harmonic functions and geometry. Finally, in Section 4 we prove Theorem 2.1 using singular solutions to the $p$-Laplace equation discovered by Aronsson and Persson.

\section{Results}

Let $B(w, \delta)=\left\{z \in \mathbf{R}^{2} ;|z-w|<\delta\right\}$ be the open disc with radius $\delta>0$ and center $w \in \mathbf{R}^{2}$ and let, for $v \in[1 / 2, \infty)$ and $p \in(1, \infty]$,

$$
q=q(v, p)=\frac{(2 v+1)(2-p)+p v^{2}+(v+1) \sqrt{(2 v+1)(2-p)^{2}+p^{2} v^{2}}}{2(p-1)(2 v+1)}
$$

interpreted as a limit when $p=\infty$, so that $q(v, \infty)=v^{2} /(2 v+1)$. 
Our main result is the following characterization of $p$-harmonic measure of plane domains. Roughly speaking, the theorem implies that if $\partial \Omega$ fits in between a sector and a generalized ball, both with aperture $\pi / v$ and apex at $w \in \partial \Omega$, then

$$
\omega_{p}\left(\Delta(w, \delta), w_{0}, \Omega\right) \approx \delta^{q},
$$

when $\delta>0$ is small. Here and in the rest of the paper, $\Delta(w, \delta)=B(w, \delta) \cap \partial \Omega$ and $A \approx B$ means that there exist constants $c$ and $C$ such that $c A \leq B \leq C A$. In general, $C$ and $c$ denote constants $C \geq 1$ and $c \leq 1$, not necessarily the same at each occurrence. Moreover, $\operatorname{dist}(x, E)$ denotes the Euclidean distance between the point $x$ and the set $E$, and a domain is an open connected set. If the domain is unbounded, then the point at infinity is by definition in the boundary. For definitions of the Harnack chain and the exterior corkscrew conditions, we refer the reader to Section 3 .

Theorem 2.1. Let $\Omega \subseteq \mathbf{R}^{2}$ be a domain, $w_{0} \in \Omega, p \in(1, \infty], v \in[1 / 2, \infty)$, $r_{1} \in(0,1)$, and let $q=q(v, p)$ be as in (2.1). There exist constants $C_{1}$ and $C_{2}$ such that the following is true.

(i) If $w \in \partial \Omega \backslash\{\infty\}$ is such that there exists a sector, with aperture $\pi / v$ and apex at $w$, which contains $\Omega$, then

$$
\omega_{p}\left(\Delta(w, \delta), w_{0}, \Omega\right) \leq C_{1}\left(\frac{\delta}{\left|w_{0}-w\right|}\right)^{q}
$$

for all $\delta>0$.

(ii) Assume that $\Omega$ satisfies the Harnack chain condition and that either $p>2$ or that $\Omega$ satisfies the exterior corkscrew condition (3.1) on $\Delta(w, \delta)$. There exists a constant $\delta_{0}>0$, such that if $w \in \partial \Omega \backslash\{\infty\}$ is of type $\left(q, r_{1}, v\right)$, then

$$
\delta^{q} \leq C_{2} \omega_{p}\left(\Delta(w, \delta), w_{0}, \Omega\right)
$$

for all $0<\delta<\delta_{0}$.

The constant $C_{1}$ depends only on $p$ and $v ; C_{2}$ depends only on $p, v, r_{1}$, $\operatorname{dist}\left(w_{0}, \partial \Omega\right)$, $\left|w-w_{0}\right|$, and if $p \in(1,2]$ also on the exterior corkscrew condition, that is, on $r_{0}$ and $M ; \delta_{0}$ depends only on $p, v, r_{1}$, and if $p \in(1,2]$ also on $r_{0}$. Moreover, $C_{1}$ decreases in $p, \delta_{0}$ increases in $p$ and, if $p>2$, then $C_{2}$ is decreasing in $p$.

From equation (4.1) in the proof of Lemma 4.1, it follows that the exponent $q=q(v, p)$ is decreasing in $p$ and increasing in $v$. Moreover, from (2.1) we obtain

$$
\lim _{p \rightarrow 1} q=\infty, \quad \lim _{p \rightarrow \infty} q=\frac{v^{2}}{2 v+1} \quad \text { and } \quad \lim _{v \rightarrow \infty} q=\infty .
$$

For the classical case $p=2$, we have that $q(v, 2)=v$, as expected.

Since the geometric assumptions in Theorem 2.1 are quite lengthy, we state the following direct consequence of Theorem 2.1 as a corollary.

Corollary 2.2. Assume that $\Omega \subseteq \mathbf{R}^{2}$ is convex and satisfies the usual interior ball condition with radius $r_{1}$. Let $w_{0} \in \Omega$ and $p \in[2, \infty]$. There exist constants $C$ and $\bar{\delta}_{0}>0$ such that

$$
C^{-1} \delta^{q(1, p)} \leq \omega_{p}\left(\Delta(w, \delta), w_{0}, \Omega\right) \leq C \delta^{q(1, p)}
$$


whenever $0<\delta<\bar{\delta}_{0}$. The constant $C$ depends only on $p, r_{1}$, $\operatorname{dist}\left(w_{0}, \partial \Omega\right)$ and $\left|w-w_{0}\right| ; \bar{\delta}_{0}$ depends only on $p$ and $r_{1}$. Moreover, if $p \geq \hat{p}>2$, then the constants $C$ and $\bar{\delta}_{0}$ can be chosen independent of $p$ but depending on $\hat{p}$.

Next, suppose that $\mathcal{S}_{v, w} \subset \mathbf{R}^{2}$ is a sector having aperture $\pi / v$ and apex at $w$. Since both the $p$-Laplace equation and $\mathcal{S}_{v, w}$ are invariant under scalings, we have, for $z \in \mathcal{S}_{v, w}$ and $s>0$

$$
\omega_{p}\left(B(w, s) \cap \partial \mathcal{S}_{v, w}, z\right)=\omega_{p}\left(B\left(w, \frac{s}{|w-z|}\right) \cap \partial \mathcal{S}_{v, w}, \frac{z}{|w-z|}\right),
$$

and hence Theorem 2.1 and Harnack's inequality implies the following.

Corollary 2.3. Let $p \in(1, \infty], v \in[1 / 2, \infty)$ and $s \in(0, \infty)$ be given. There exists a constant $C$, depending only on $p$ and $v$, such that

$$
C^{-1}\left(\frac{s}{|w-z|}\right)^{q} \leq \omega_{p}\left(B(w, s) \cap \partial \mathcal{S}_{v, w}, z, \mathcal{S}_{v, w}\right) \leq C\left(\frac{s}{|w-z|}\right)^{q}
$$

whenever $z \in \mathcal{S}_{2 v, w} \backslash B(w, s)$.

In the case $p=\infty$ we have the following extension of Theorem 2.1 to $\mathbf{R}^{n}$. When $\Omega$ is a unit ball, this result was first proved in [PSSW09, Theorem 1.5].

Corollary 2.4. Assume that $\Omega \subseteq \mathbf{R}^{n}$ is rotationally invariant around an axis $\ell$. Let $\Omega_{\ell}$ be the intersection between $\Omega$ and a two-dimensional plane containing $\ell$, let $w_{0} \in \Omega, v \in[1 / 2, \infty)$ and $r_{1} \in(0,1)$. There exist constants $\widehat{C}_{1}$ and $\widehat{C}_{2}$ such that the following is true.

(i) If $w \in \partial \Omega \cap \ell$ is such that there exists a sector with aperture $\pi / v$ and apex at $w$ that contains $\Omega_{\ell}$, then

$$
\omega_{\infty}\left(\Delta(w, \delta), w_{0}, \Omega\right) \leq \widehat{C}_{1}\left(\frac{\delta}{\left|w_{0}-w\right|}\right)^{\frac{v^{2}}{2 v+1}}
$$

for all $\delta>0$.

(ii) Assume that $\Omega$ satisfies the Harnack chain condition. There exists a constant $\widehat{\delta}_{0}>0$, such that if $w \in \partial \Omega \cap \ell$ is of type $\left(v^{2} /(2 v+1), r_{1}, v\right)$, then

$$
\delta^{\frac{v^{2}}{2 v+1}} \leq \widehat{C}_{2} \omega_{\infty}\left(\Delta(w, \delta), w_{0}, \Omega\right)
$$

for all $0<\delta<\widehat{\delta}_{0}$.

The constant $\widehat{C}_{1}$ depends only on $v ; \widehat{C}_{2}$ depends only on $v, r_{1}$, $\operatorname{dist}\left(w_{0}, \partial \Omega\right)$ and $\left|w-w_{0}\right|$; while $\widehat{\delta}_{0}$ depends only on $v$ and $r_{1}$.

To prove Corollary 2.4, assume that $u \in C^{2}(\Omega)$, otherwise, we switch to a $C^{2}$ function through the definition of viscosity solutions. Since the $\infty$-Laplacian is invariant under rotations and translations, we also assume that the axis $\ell$ coincides with the $x_{1}$-axis and that $\Omega_{\ell}$ is contained in the $x_{1} x_{2}$-plane. By symmetry, we conclude that $u_{x_{3}}=u_{x_{4}}=\cdots=u_{x_{n}}=0$ on $\Omega_{\ell}$ and hence

$$
\Delta_{\infty} u=\sum_{i, j=1}^{n} u_{x_{i}} u_{x_{j}} u_{x_{i} x_{j}}=u_{x_{1}}^{2} u_{x_{1} x_{1}}+2 u_{x_{1}} u_{x_{2}} u_{x_{1} x_{2}}+u_{x_{2}}^{2} u_{x_{2} x_{2}}=0 .
$$

Thus, $u$ is $\infty$-harmonic in $\Omega_{\ell} \subseteq \mathbf{R}^{2}$, which allows us to deduce Corollary 2.4 from Theorem 2.1 . 
Returning to two dimensions, let $G_{p}$ be the $p$-Green function for $\Omega \subset \mathbf{R}^{2}$, that is, a weak solution to

$$
\begin{cases}\Delta_{p} G_{p}(\cdot, z)=-\delta_{z} & \text { in } \Omega, \\ G_{p}(\cdot, z)=0 & \text { on } \partial \Omega,\end{cases}
$$

such that $G_{p}(w, z) \cdot|w-z|^{(2-p) /(p-1)}$ is bounded, and let $K_{p}$ be the $p$-Martin kernel, defined in the usual way; see [Hir08] for details.

Corollary 2.5. Let $p \in(1, \infty]$, let $\Omega$ be a bounded $C^{1,1}$-domain in $\mathbf{R}^{2}$ and let $w_{0} \in \Omega$ be fixed. Then there exist a point $\widehat{w} \in \partial \Omega$ and a constant $C$ such that

$$
\omega_{p}\left(\Delta(\widehat{w}, \delta), w_{0}, \Omega\right) \leq C \delta^{q(1, p)}
$$

for $\delta>0$ small enough. The constant $C$ depends only on $p$.

In fact, convexity must hold in some point $\widehat{w} \in \partial \Omega$, and hence Theorem 2.1 yields the result. In connection with Hirata, we give the following remark.

Remark 2.6. For a $C^{1,1}$-domain in $\mathbf{R}^{n}$ and for $p \in(1, n]$, Hirata [Hir08, Theorem 2.7] proved that the following statements are equivalent to each other. Let $r_{1}$ be the radius in the ball condition (interior and exterior) guaranteed by [AKSZ07, Lemma 2.2], and let $x_{0} \in \Omega$.

(a) $\omega_{p}\left(\Delta(w, r), x_{0}\right) \approx r^{(n-1) /(p-1)}$ whenever $w \in \partial \Omega$ and $0<r<r_{1}$,

(b) $G_{p}(x, y) \approx G_{p}(y, x)$ for any pair $x, y \in \Omega$,

(c) $G_{p}\left(x_{0}, x\right) \approx \operatorname{dist}(x, \partial \Omega)$ whenever $\operatorname{dist}(x, \partial \Omega)<r_{1}$,

(d) for each $w \in \partial \Omega$, there is a positive $p$-harmonic function $u$ on $\Omega$ vanishing continuously on $\partial \Omega \backslash\{w\}$ such that $u\left(x_{0}\right)=1$ and $u(x) \approx \operatorname{dist}(x, \partial \Omega)^{(1-n) /(p-1)}$ on the inward normal at $w$,

(e) for each $w \in \partial \Omega, K_{p}(x, w) \approx \operatorname{dist}(x, \partial \Omega) /|x-w|^{(n+p-2) /(p-1)}$ for $x \in \Omega$.

Hirata then proved (d) for $p=n$; and hence all of (a)-(e) are valid for $p=n$. It is classical that all of (a)-(e) are valid for the linear case $p=2$. The validity for the other case is not known. Corollary 2.5 implies that none of (a)-(e) holds for $1<p<2$ and $n=2$, since $1 /(p-1)<q(1, p)$.

In the linear case $p=2$, it is well known that an upper bound on the harmonic measure of discs immediately gives a lower bound on the Hausdorff dimension of the harmonic measure. More precisely, if $\omega_{2}\left(\Delta(w, \delta), w_{0}\right) \leq C \delta^{v}$, for all $w \in \partial \Omega$ and all sufficiently small $\delta>0$, then the Hausdorff dimension satisfies $\operatorname{dim}_{H}(E) \geq v$, for all $E \subseteq \partial \Omega$ with $\omega_{2}\left(E, w_{0}\right)>0$. In passing, we recall that Beurling's projection theorem gives the lower bound $\operatorname{dim}_{H}(E) \geq 1 / 2$ for all simply connected domains [GM05, Corollary III.9.3]. This bound was improved by Carleson [Car73] to $1 / 2+c$, with $c>0$ a universal constant, and Makarov [Mak85] proved, in particular, that $\operatorname{dim}_{H}(E)=1$ for Jordan domains.

When $p \neq 2$, however, the $p$-harmonic measure is in general not subadditive, not even if we allow a multiplicative factor. For instance, it is known that the boundary of the half-plane can be partitioned into finitely many sets of $p$-harmonic measure zero [LMW05]. Although Theorem 2.1 shows that the pointwise dimension, defined in the obvious way, is $q=q(v, p)$, there are no immediate ties between this and the dimension of the $p$-harmonic measure. For the question of the dimension of a different notion of $p$-harmonic measure, coinciding with $\omega_{p}$ only when $p=2$, we refer to [BL05]. 
Concerning doubling properties of the $p$-harmonic measure we note the following. Theorem 2.1 implies the existence of a constant $C$ such that

$$
\omega_{p}\left(\Delta(w, 2 \delta), w_{0}\right) \leq C \omega_{p}\left(\Delta(w, \delta), w_{0}\right),
$$

if $\delta$ is small enough. Unfortunately, $C$ depends on both $w$ on $w_{0}$. If we in addition assume that the boundary conditions imposed on $w$ holds uniformly for all $w \in \partial \Omega$, then $C$ can be chosen independent of $w$. We note that such assumption implies that $v=1$ and hence $\Omega$ is a convex domain which satisfies the generalized interior ball condition. If also $p=2$, then $\Omega$ satisfies the usual interior ball condition, and then $\Omega$ is a $C^{1,1}$-domain, see [AKSZ07, Lemma 2.2].

Acknowledgement. The authors want to thank G. Aronsson, J. Björn, T. Lundh and K. Nyström for useful discussions and ideas. We also thank the anonymous referee for a careful reading of the paper and for constructive comments.

\section{Preliminaries}

In this section we state some definitions and known results for $p$-harmonic measure and $p$-harmonic functions. We start with defining solutions and $p$-harmonicity. If $p \in(1, \infty)$, we say that $u$ is a (weak) subsolution (supersolution) to the $p$-Laplacian in a domain $\Omega$ provided $u \in W_{l o c}^{1, p}(\Omega)$ and

$$
\int_{\Omega}|\nabla u|^{p-2}\langle\nabla u, \nabla \theta\rangle d x \leq(\geq) 0,
$$

whenever $\theta \in C_{0}^{\infty}(\Omega)$ is non-negative. A function $u$ is a (weak) solution of the $p$-Laplacian if it is both a subsolution and a supersolution. Here, as in the sequel, $W^{1, p}(\Omega)$ is the Sobolev space of those $p$-integrable functions whose first distributional derivatives are also $p$-integrable, and $C_{0}^{\infty}(\Omega)$ is the set of infinitely differentiable functions with compact support in $\Omega$. If $p=\infty$, the equation is no longer of divergence form and therefore the above definition is replaced by the following. Here, as in the sequel, $\Delta_{\infty}$ is the $\infty$-Laplace operator defined in (1.1).

An upper semicontinuous function $u: \Omega \rightarrow \mathbf{R}$ is a (viscosity) subsolution of the $\infty$-Laplacian in $\Omega$ provided that for each function $\psi \in C^{2}(\Omega)$ such that $u-\psi$ has a local maximum at a point $x_{0} \in \Omega$, we have $\Delta_{\infty} \psi\left(x_{0}\right) \geq 0$. A lower semicontinuous function $u: \Omega \rightarrow \mathbf{R}$ is a (viscosity) supersolution of the $\infty$-Laplacian in $\Omega$ provided that for each function $\psi \in C^{2}(\Omega)$ such that $u-\psi$ has a local minimum at a point $x_{0} \in \Omega$, we have $\Delta_{\infty} \psi\left(x_{0}\right) \leq 0$. A function $u: \Omega \rightarrow \mathbf{R}$ is a (viscosity) solution of the $\infty$-Laplacian if it is both a subsolution and a supersolution.

If $u$ is an upper semicontinuous subsolution to the $p$-Laplacian in $\Omega, p \in(1, \infty]$ then we say that $u$ is $p$-subharmonic in $\Omega$. If $u$ is a lower semicontinuous supersolution to the $p$-Laplacian in $\Omega, p \in(1, \infty]$, then we say that $u$ is $p$-superharmonic in $\Omega$. If $u$ is a continuous solution to the $p$-Laplacian in $\Omega, p \in(1, \infty]$, then $u$ is $p$-harmonic in $\Omega$.

We note that for the $p$-Laplacian, $1<p<\infty$, weak solutions are also viscosity solutions (defined as above but with $\Delta_{\infty}$ replaced by $\Delta_{p}$ ); see [Ju98, Theorem 1.29]. Moreover, under suitable assumptions, an $\infty$-harmonic function is the uniform limit of a sequence of $p$-harmonic functions as $p \rightarrow \infty$; see [J93]. For more on weak solutions, viscosity solutions, $p$-harmonicity and $p$-superharmonicity, see for instance [HKM93] and [CIL92]. 
Concerning geometric assumptions, we recall the following. A domain $\Omega$ is said to satisfy the Harnack chain condition, if given any $\epsilon>0$ and $w_{1}, w_{2} \in \Omega$ such that

$$
\operatorname{dist}\left(w_{1}, \partial \Omega\right)>\epsilon, \quad \operatorname{dist}\left(w_{2}, \partial \Omega\right)>\epsilon \quad \text { and } \quad\left|w_{1}-w_{2}\right|<C \epsilon
$$

for some constant $C$, then there exists a Harnack chain from $w_{1}$ to $w_{2}$ whose length may depend on $C$, but not on $\epsilon$.

A domain $\Omega$ is said to satisfy the exterior corkscrew condition on $E \subset \partial \Omega$ with constants $r_{0}$ and $M>1$, if the following holds. For any $w \in E$ and any $r \in\left(0, r_{0}\right)$, there exists a point $a_{r}(w) \in \mathbf{R}^{n} \backslash \Omega$ such that

$$
\frac{r}{M}<\left|a_{r}(w)-w\right|<r \quad \text { and } \operatorname{dist}\left(a_{r}(w), \partial \Omega\right)>\frac{r}{M} .
$$

As mentioned in the Introduction, the $p$-harmonic measure $\omega_{p}(E, \cdot)$ is a $p$ harmonic function $\Omega \rightarrow[0,1]$, and the following lemma tells us that the boundary behaviour at $p$-regular boundary points (regular with respect to the $p$-Laplace operator) is as expected.

Lemma 3.1. Let $p \in(1, \infty]$, let $z_{0}$ be a $p$-regular boundary point of a domain $\Omega \subset \mathbf{R}^{n}$, and let $E \subseteq \partial \Omega$. If $z_{0}$ has a neighborhood $V$ such that $V \cap \partial \Omega \subseteq E$, then

$$
\lim _{z \rightarrow z_{0}} \omega_{p}(E, z, \Omega)=1 \text {. }
$$

Similarly, if $z_{0}$ has a neighborhood $V$ such that $V \cap \partial \Omega \cap E=\emptyset$, then

$$
\lim _{z \rightarrow z_{0}} \omega_{p}(E, z, \Omega)=0 .
$$

Proof. In the case $p \in(1, \infty)$ this follows from [HKM93, Theorem 11.6]. If $p=\infty$, then the result folows by definition, see [PSSW09, pp. 173-174].

Recall that a sufficient condition for $p$-regularity is that the domain satisfies the exterior corkscrew condition, as defined in (3.1). See [HKM93, Theorem 6.31].

We will make use of the comparison principle and Harnack's inequality for $p$ harmonic functions, as well as the fact that these results hold with constants independent of $p$, if $p$ is large.

Lemma 3.2. Let $p \in(1, \infty]$ and suppose that $u$ is $p$-superharmonic and that $v$ is $p$-subharmonic in a bounded domain $\Omega \subset \mathbf{R}^{n}$. If

$$
\limsup _{z \rightarrow w} v(z) \leq \liminf _{z \rightarrow w} u(z)
$$

for all $w \in \partial \Omega$, and if both sides of the above inequality are not simultaneously $\infty$ or $-\infty$, then $v \leq u$ in $\Omega$.

Proof. If $p \in(1, \infty)$, this follows from [HKM93, Theorem 7.6]. For the case $p=\infty$, this was first proved in [J93, Theorem 3.11]. A shorter proof was later presented in [AS10].

Lemma 3.3. Let $p \in(1, \infty], w \in \mathbf{R}^{2}, r \in(0, \infty)$ and suppose that $u$ is a nonnegative $p$-harmonic function in $B(w, 2 r)$. Then there exists a constant $C \in[1, \infty)$, depending only on $p$, such that

$$
\sup _{B(w, r)} u \leq C \inf _{B(w, r)} u .
$$

Moreover, if $p>2$, then the constant $C$ is decreasing in $p$. 
Proof. For the case $p \in(1, \infty)$, see [KMV96] or [LuN10, Lemma 2.3]. For the case $p=\infty$ the result follows by taking the limit $p \rightarrow \infty$ in the former case, see [LM95].

Classically, every harmonic function $u$ in a simply connected plane domain has a harmonic conjugate, that is, a harmonic function $v$ such that $u_{x}^{\prime}=v_{y}^{\prime}$ and $u_{y}^{\prime}=-v_{x}^{\prime}$. For general $p$-harmonic functions, $p \in(1, \infty)$, the corresponding concept is that of a stream function.

Definition 3.4. Let $p \in(1, \infty)$ and denote by $p^{\prime}=p /(p-1)$ the conjugate index. If $u$ is $p$-harmonic, a $p^{\prime}$-harmonic function $v$ is said to be a stream function to $u$, if $u_{x}^{\prime}=|\nabla v|^{p^{\prime}-2} v_{y}^{\prime}$ and $u_{y}^{\prime}=-|\nabla v|^{p^{\prime}-2} v_{x}^{\prime}$.

Similar to the classical case, every $p$-harmonic function in a simply connected plane domain has a $p^{\prime}$-harmonic stream function, see [AL88, Theorem 4].

\section{Proofs}

To prove Theorem 2.1, we use similar ideas as in the proof of [PSSW09, Theorem 1.5], which is the corresponding result for $\infty$-harmonic measure in any dimension $n \geq 2$, when the domain is the unit ball. For $p \geq 2$ we use a $p$-harmonic function from [Aro86], and if $p<\infty$, then the function has a $p /(p-1)$-harmonic stream function which - making use of a representation from [Per89] - also has the desired properties. The functions are defined in the sector $\mathcal{S}_{v}$ and can be written

$$
H_{v, p}(x, y)=r^{-q} h_{v, p}(\phi),
$$

where $h_{v, p}$ and $q=q(v, p)$ are certain functions given below, and $(r, \phi)$ are polar coordinates for $(x, y)$. We summarize some properties of these functions in the following lemma.

Lemma 4.1. Let $p \in(1, \infty]$ and let $v \in\left[\frac{1}{2}, \infty\right)$. There exists a $p$-harmonic function $H_{v, p}: \mathcal{S}_{v} \rightarrow(0, \infty)$ of the form $H_{v, p}(x, y)=r^{-q} h_{v, p}(\phi)$, where $q=q(v, p)$ is given by (2.1). The exponent $q$ is decreasing in $p$ and increasing in $v$. Moreover, the function $h_{v, p}$ is continuous on $\left[-\frac{\pi}{2 v}, \frac{\pi}{2 v}\right]$, differentiable, and satisfies

(i) $h_{v, p}\left( \pm \frac{\pi}{2 v}\right)=0$,

(ii) $h_{v, p}(\phi),\left|h_{v, p}^{\prime}(\phi)\right| \leq M_{v, p}<\infty$, for all $\phi \in\left(-\frac{\pi}{2 v}, \frac{\pi}{2 v}\right)$, and

(iii) $\min _{|\phi| \leq \frac{\pi}{4 v}} h_{v, p}(\phi) \geq m_{v}>0$,

where $M_{v, p}$ and $m_{v}$ are constants depending only on $v, p$ and $v$, respectively. Moreover $M_{v, p}$ is decreasing in $p$ with $v$ fixed, and $M_{v, p} \leq v$ for $p \geq 2$.

For the convenience of a reader who has access to [Aro86] but not [Per89], we record the following result from [Per89], which allows the results in [Aro86] to be extended to $p \in(1,2)$.

Lemma 4.2. Assume that $p \in(2, \infty)$ and that $q>0$. Let $p^{\prime}=p /(p-1)$ be the conjucate index to $p$, and define $q^{\prime}$ by the relation $(1+q) p=\left(1+q^{\prime}\right) p^{\prime}$. If $(r, \phi) \mapsto r^{-q} h(\phi)$ is $p$-harmonic in a sector $\mathcal{S}_{v}$, then

$$
(r, \phi) \mapsto r^{-q^{\prime}} \cdot \frac{1}{q^{\prime}}\left((q h(\phi))^{2}+\left(h^{\prime}(\phi)\right)^{2}\right)^{\frac{p-2}{2}} h^{\prime}(\phi)
$$

is a $q^{\prime}$-harmonic stream function in $\mathcal{S}_{v}$. 
In [Per89, Theorem 1A], this is proved by observing that the condition [Aro86, Equation (5)] for $p$-harmonicity of $(r, \phi) \mapsto r^{-q} h(\phi)$, also for $p \in(1,2)$, can be written [Per89, Equation (3.3)] as

$$
\frac{\mathrm{d}}{\mathrm{d} \phi}\left(h^{\prime} \cdot\left((q h)^{2}+\left(h^{\prime}\right)^{2}\right)^{\frac{p-2}{2}}\right)+q q^{\prime} h \cdot\left((q h)^{2}+\left(h^{\prime}\right)^{2}\right)^{\frac{p-2}{2}}=0 .
$$

To shorten our proof, we will also make use of a compact representation formula given by [Per89] of the stream function, see Case 3 in the proof of Lemma 4.1. Of crucial importance, however, is the fact that the correct radial exponent $q^{\prime}$ is given by the relation $(1+q) p=\left(1+q^{\prime}\right) p^{\prime}$.

Proof of Lemma 4.1. The facts (ii) and (iii) are actually quite easy to see from continuity and positivity of $h_{v, p}(\phi)$. However, the proof of Theorem 2.1 calls for better control of the constants in the lemma, which requires the following more delicate proof.

For $p=2$, we immediately see that $H_{v, p}(x, y)=r^{-v} \cos (v \phi)$ is a function with the desired properties, since $q(v, 2)=v$. Next, we prove the lemma for $p \in(2, \infty]$, and then show that $H_{v, p}$, when $p<\infty$, determines a stream function which is the required function for $p /(p-1) \in(1,2)$.

Case 1: $2<p<\infty$. By [Aro86, Case 2 on p. 145], there exists a $p$-harmonic function $H_{v, p}$, defined in $\mathcal{S}_{v}$, of the form $H_{v, p}(x, y)=r^{-q} h_{v, p}(\phi)$. The function $h_{v, p}$ can be written as

$$
h_{v, p}(\phi)=\cos \theta_{v, p}(\phi) \cdot\left(1+\frac{\cos ^{2} \theta_{v, p}(\phi)}{a q}\right)^{-\frac{q+1}{2}}
$$

where $a=(p-1) /(p-2)$ and $\theta_{v, p}$ is a certain continuous, strictly decreasing function of $\phi$.

When $|\phi|<\frac{\pi}{2 v}$, we have that

$$
\phi=\theta_{v, p}(\phi)-\frac{v+1}{v} \arctan \left(\mu_{v, p} \tan \theta_{v, p}(\phi)\right), \quad \text { where } \mu_{v, p}=\sqrt{\frac{a q}{a q+1}},
$$

see [Aro86, p. 145]. The function $\theta_{v, p}$ is chosen so that it maps the interval $\left[-\frac{\pi}{2 v}, \frac{\pi}{2 v}\right]$ to $\left[-\frac{\pi}{2}, \frac{\pi}{2}\right]$, and this condition immediately determines the radial exponent $q$.

More precisely, the condition that determines $q$ is given by [Aro86, p. 146], and reads

$$
\frac{\pi}{v}=-\phi\left(\frac{\pi}{2}\right)+\phi\left(-\frac{\pi}{2}\right)=\pi \sqrt{\frac{a q}{a q+1}}\left(1+\frac{1}{q}\right)-\pi
$$

This equation in $q$ was derived and solved in [Aro86, Equation (8)] for $v$ an integer, but that assumption is needed only when the function is extended to the entire plane. Solving the equation first for $a=(p-1) /(p-2)$ and then for $p$, we obtain

$$
p=1+\frac{1}{1+q}+\frac{v^{2}}{(2 v+1) q-v^{2}} .
$$

From (4.1) we see that $q$ is decreasing in $p$ and increasing in $v$. Solving for $q$, we arrive at a quadratic equation, whose unique positive root $q=q(v, p)$ is given by (2.1) in the Introduction.

We proceed by observing that $\mu_{v, p}$ is strictly decreasing in $p$, since $p>2$ and $q$ is decreasing in $p$, with $\lim _{p \rightarrow \infty} \mu_{v, p}=v /(v+1)$ and $\lim _{p \rightarrow 2} \mu_{v, p}=1$, so that 
$\mu_{v, p} \in\left(\frac{v}{v+1}, 1\right)$. It is then easy to see that $\min _{|\phi| \leq \frac{\pi}{4 v}} h_{v, p}(\phi) \geq c$, for some constant $c>0$ independent of $p \in(2, \infty)$, since $\theta_{v, p}(\phi)$ is bounded away from $\pm \frac{\pi}{2}$, uniformly in $p$, if $|\phi| \leq \frac{\pi}{4 v}$ and $v \in\left[\frac{1}{2}, \infty\right)$ is fixed.

Next, note that $0 \leq h_{v, p}(\phi) \leq 1$ for $|\phi| \leq \frac{\pi}{2 v}$, and that $h_{v, p}\left( \pm \frac{\pi}{2 v}\right)=0$. Finally, using the formula [Aro86, page 143]

$$
h_{v, p}^{\prime}(\phi)=q \cdot \sin \theta_{v, p}(\phi) \cdot\left(1+\frac{\cos ^{2} \theta_{v, p}(\phi)}{a q}\right)^{-\frac{q+1}{2}},
$$

we have that $\left|h_{v, p}^{\prime}(\phi)\right| \leq q$, for all $\phi \in\left[-\frac{\pi}{2 v}, \frac{\pi}{2 v}\right]$, and hence also that $\left|h_{v, p}^{\prime}(\phi)\right| \leq v$, since $q(v, p) \leq q(v, 2)=v$. The proof of the lemma in the case when $p \in(2, \infty)$ is complete.

Case 2: $p=\infty$. Letting $a=1$ when $p=\infty$, the function from Case 1 is immediately extended to the case when $p=\infty$. By [Aro86, Case 2 on p. 148] and [Aro86, Lemma $\left.3^{\prime}\right]$, this function is smooth and $\infty$-harmonic when $\phi \neq 0$. Consequently, the function $H_{v, \infty}$ satisfies the required conditions, except that it is not immediate that the function is $\infty$-harmonic in the viscosity sense in the entire sector.

For $v=1$, it was shown in [Bha04, Appendix I] that $H_{1, \infty}$ is indeed $\infty$-harmonic in the viscosity sense. However, the exact same proof works for all $v \geq \frac{1}{2}$; or more generally for $\infty$-harmonic functions with polar representation $r^{-q} h(\phi) \geq 0$, with $q \cdot(q+1) \geq 0$ and where $h \in C^{1}$ is $C^{2}$ for $\phi \neq 0$, has a local maximum at $\phi=0$ and satisfies $\lim _{\phi \rightarrow 0} h^{\prime \prime}(\phi)=-\infty$. Thus the proof of the lemma is complete in the case when $p=\infty$.

Case 3: $1<p<2$. It is convenient to view the $p$-values at hand as conjugate to those in Case 1 . Hence, let $\epsilon \in(0,1)$, fix $p^{\prime} \in(1+\epsilon, 2)$, and denote the conjugate index by $p=p^{\prime} /\left(p^{\prime}-1\right)$.

First we extend the domain of $h_{v, p}$, defined in Case 1 , to $\mathbf{R}$. To do so, we first extend $\theta_{v, p}$ by 'pasting' together copies of its graph on $\left[-\frac{\pi}{2 v}, \frac{\pi}{2 v}\right]$, so that, for instance, $\theta_{v, p}(\phi)=\pi+\theta_{v, p}\left(\phi+\frac{\pi}{v}\right)$ for $\phi \in\left[-\frac{\pi}{v},-\frac{\pi}{2 v}\right]$, which is the extension we will need. Using this extension of $\theta_{v, p}$, the domain of definition of $h_{v, p}$ is extended to $\mathbf{R}$, and although the function is no longer positive at all points, we still have that $\left|h_{v, p}\right| \leq 1$ and $\left|h_{v, p}^{\prime}\right| \leq q$.

By [Aro86, Case 2 on p. 145], restricting $(r, \phi) \mapsto r^{-q} h_{v, p}(\phi), r>0, \phi \in \mathbf{R}$, to any angular interval of length $2 \pi$ or less yields a $p$-harmonic function. In particular, let $\bar{H}_{v, p}(x, y)=r^{-q} h_{v, p}(\phi)$ for $r>0$ and $\phi \in\left[-\frac{\pi}{v}, 0\right]$, which is then $p$-harmonic for $r>0$ and $\phi \in\left(-\frac{\pi}{v}, 0\right)$. As we shall see, the stream function of $\bar{H}_{v, p}$ is, up to rotation, the desired function.

Let $F_{v, p^{\prime}}(x, y)=r^{-q\left(v, p^{\prime}\right)} f_{v, p^{\prime}}(\phi)$, for $r>0$ and $\phi \in\left[-\frac{\pi}{v}, 0\right]$, where

$$
f_{v, p^{\prime}}(\phi)=\sin \theta_{v, p}(\phi) \cdot\left(1-\frac{\left(2-p^{\prime}\right) \sin ^{2} \theta_{v, p}(\phi)}{\left(p^{\prime}-1\right) q\left(v, p^{\prime}\right)}\right)^{-\frac{q\left(v, p^{\prime}\right)+1}{2}} .
$$

Here $q\left(v, p^{\prime}\right)$ satisfies

$$
(1+q(v, p)) p=\left(1+q\left(v, p^{\prime}\right)\right) p^{\prime}
$$

as can be verified by plugging in $p^{\prime}=\frac{p}{p-1}$ and then expanding the expression using the definition of $q$, formula (2.1). Since, by a calculation, $\frac{2-p^{\prime}}{\left(p^{\prime}-1\right) q\left(\frac{1}{2}, p^{\prime}\right)} \leq \frac{8}{9}$ and $q\left(\frac{1}{2}, p^{\prime}\right) \leq$ $q\left(v, p^{\prime}\right) \leq q(v, 1+\epsilon)$, we see that $\left|f_{v, p^{\prime}}(\phi)\right| \leq C$, for a constant $C$ depending only on 
$\epsilon$ and $v$. Then by [Per89, Theorem 2], as can also be verified using Lemma 4.2 and (4.2), $F_{v, p^{\prime}}$ is a stream function to the function $\gamma_{v, p} \bar{H}_{v, p}$, where

$$
\gamma_{v, p}=\frac{q\left(v, p^{\prime}\right)^{\frac{1}{p-1}}}{q(v, p)}\left(\frac{q\left(v, p^{\prime}\right)}{(p-1) q(v, p)}\right)^{\frac{q(v, p)+1}{2}}
$$

is a constant, and we see that $\gamma_{v, p} \leq C$, for all $p^{\prime} \in(1+\epsilon, 2)$ and $p=p^{\prime} /\left(p^{\prime}-1\right)$, where $C$ only depends on $\epsilon$ and $v$.

Since $\bar{H}_{v, p}$ is $p$-harmonic for $r>0$ and $\phi \in\left(-\frac{\pi}{v}, 0\right)$, it follows that $F_{v, p^{\prime}}$ is $p^{\prime}$ harmonic in the same domain, and that $\left|\nabla F_{v, p^{\prime}}\right|=\gamma_{v, p}^{p-1} \cdot\left|\nabla \bar{H}_{v, p}\right|^{p-1}$. Hence, writing the gradient in polar coordinates and simplifying, we get that

$$
\left|f_{v, p^{\prime}}^{\prime}(\phi)\right| \leq \gamma_{v, p}^{p-1}\left(\left(-q(v, p) h_{v, p}(\phi)\right)^{2}+\left(h_{v, p}^{\prime}(\phi)\right)^{2}\right)^{\frac{p-1}{2}}
$$

where we have used the fact that $(q(v, p)+1)(p-1)=q\left(v, p^{\prime}\right)+1$. Since $\left|h_{v, p}\right| \leq 1$ and $\left|h_{v, p}^{\prime}\right| \leq q(v, p)$, we get that $\left|f_{v, p^{\prime}}^{\prime}(\phi)\right| \leq C$, for all $\phi \in\left[-\frac{\pi}{v}, 0\right]$ and all $p^{\prime} \in(1+\epsilon, 2)$, for some constant $C$ depending only on $\epsilon$ and $v$.

If $v \in\left[\frac{1}{2}, \infty\right)$ is fixed, then $\theta_{v, p}(\phi)$ is uniformly bounded away from 0 and $\pi$ when $\phi \in\left[-\frac{3 \pi}{4 v},-\frac{\pi}{4 v}\right]$ and $p^{\prime} \in(1,2)$. Hence there exists a constant $c>0$, independent of $p^{\prime} \in(1,2)$, such that $f_{v, p^{\prime}}(\phi) \geq c$ for all $\phi \in\left[-\frac{3 \pi}{4 v},-\frac{\pi}{4 v}\right]$. We also see that $f_{v, p^{\prime}}(\phi) \geq 0$, all $\phi \in\left[-\frac{\pi}{v}, 0\right]$, and that $f_{v, p^{\prime}}(0)=f_{v, p^{\prime}}\left(-\frac{\pi}{v}\right)=0$. Letting $h_{v, p^{\prime}}(\phi)=f_{v, p^{\prime}}\left(\phi+\frac{\pi}{2 v}\right)$, for $\phi \in\left[-\frac{\pi}{v}, 0\right]$, we see that $H_{v, p^{\prime}}=r^{-q\left(v, p^{\prime}\right)} h_{v, p^{\prime}}(\phi)$ is the desired function. The proof of Lemma 4.1 is complete.

Proof of Theorem 2.1. Part (i). Fix $p \in(1, \infty], v \in\left[\frac{1}{2}, \infty\right)$, and $w \in \partial \Omega \backslash\{\infty\}$. After possibly translating and rotating, we may assume that $w=(0,0)$ and that $\Omega \subseteq \mathcal{S}_{v}$, where $\mathcal{S}_{v}$ is the sector defined in (1.2). To simplify notation, we also put $A_{\delta}=\Delta((0,0), \delta)=B((0,0), \delta) \cap \partial \Omega$, for $\delta>0$.

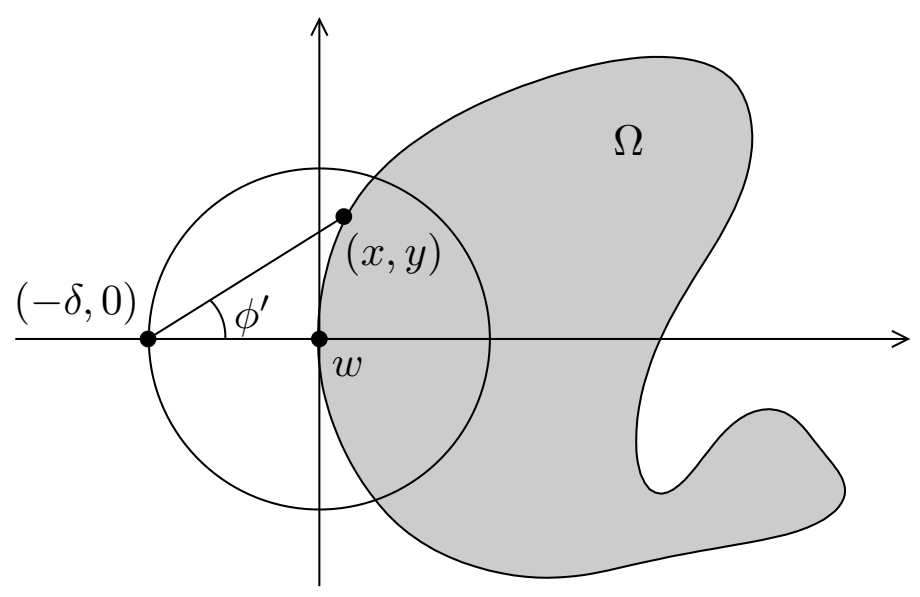

Figure 2. Geometry for the upper bound on $\omega_{p}\left(A_{\delta}, w_{0}\right)$, part (i) of Theorem 2.1. The function $\widetilde{H}_{\delta}$ has a pole at $(-\delta, 0)$, and is shown to be bounded from below by some constant on $A_{\delta}$. In the figure $v=1$, so that $\Omega$ is contained in a half-plane.

Let $\widetilde{H}_{\delta}(x, y)=(2 \delta)^{q} H_{v, p}(x+\delta, y)$, for $(x, y) \in \mathcal{S}_{v}$, where $H_{v, p}$ is the function from Lemma 4.1. If $(x, y) \in A_{\delta}$, then we see geometrically that the polar angle $\phi^{\prime}$ of the point $(x+\delta, y)$ satisfies $\left|\phi^{\prime}\right| \leq \frac{\pi}{4 v}$, since $\phi^{\prime}$ is no larger than the inscribed angle of an arc with central angle $\frac{\pi}{2 v}$ (Figure 2). This fact, which trivially holds if $v=\frac{1}{2}$, can 
also be seen algebraically, for $v>\frac{1}{2}$, by letting $(r, \phi)$ be polar coordinates for $(x, y)$, and noting that

$$
\cot \left|\phi^{\prime}\right|=\frac{x+\delta}{|y|}=\frac{\cos |\phi|+\frac{\delta}{r}}{\sin |\phi|} \geq \cot \frac{\pi}{2 v}+\csc \frac{\pi}{2 v}=\cot \frac{\pi}{4 v},
$$

since $r \leq \delta$ and $|\phi| \leq \frac{\pi}{2 v}$. Hence, we may use property (iii) in Lemma 4.1 to get that, for $(x, y) \in A_{\delta}$,

$$
\widetilde{H}_{\delta}(x, y) \geq(2 \delta)^{q} \cdot m_{v} \cdot(2 \delta)^{-q}=m_{v},
$$

since $A_{\delta} \subseteq B((-\delta, 0), 2 \delta)$, implying that $1_{\overline{A_{\delta}}} \leq \widetilde{H}_{\delta} / m_{v}$ on $\partial \Omega$. By the definition of $p$-harmonic measure (Definition 1.1), this implies that, for all $w_{0}=\left(x_{0}, y_{0}\right) \in \Omega$,

$\omega_{p}\left(A_{\delta}, w_{0}\right) \leq \frac{(2 \delta)^{q}}{m_{v}} \cdot H_{v, p}\left(x_{0}+\delta, y_{0}\right) \leq \frac{(2 \delta)^{q} M_{v, p}}{m_{v}} \cdot\left|\left(x_{0}+\delta, y_{0}\right)\right|^{-q} \leq \frac{2^{q} M_{v, p}}{m_{v}} \cdot \frac{\delta^{q}}{\left|w_{0}-w\right|^{q}}$,

allowing us to pick $C_{1}=2^{q} M_{v, p} / m_{v}$.

Finally, since $M_{v, p}$ and $q=q(v, p)$ are decreasing in $p$, we can conclude that $C_{1}$ is decreasing in $p$, completing the proof of part (i) of Theorem 2.1.

Part (ii). Fix $p \in(1, \infty], v \in\left[\frac{1}{2}, \infty\right)$, and $w \in \partial \Omega \backslash\{\infty\}$. After possibly translating and rotating, we may assume that $w=(0,0)$ and that $E\left(q, r_{1}, v\right) \subseteq \Omega$ (Figure 3), where $E\left(q, r_{1}, v\right)$ is the set in Definition 1.2.

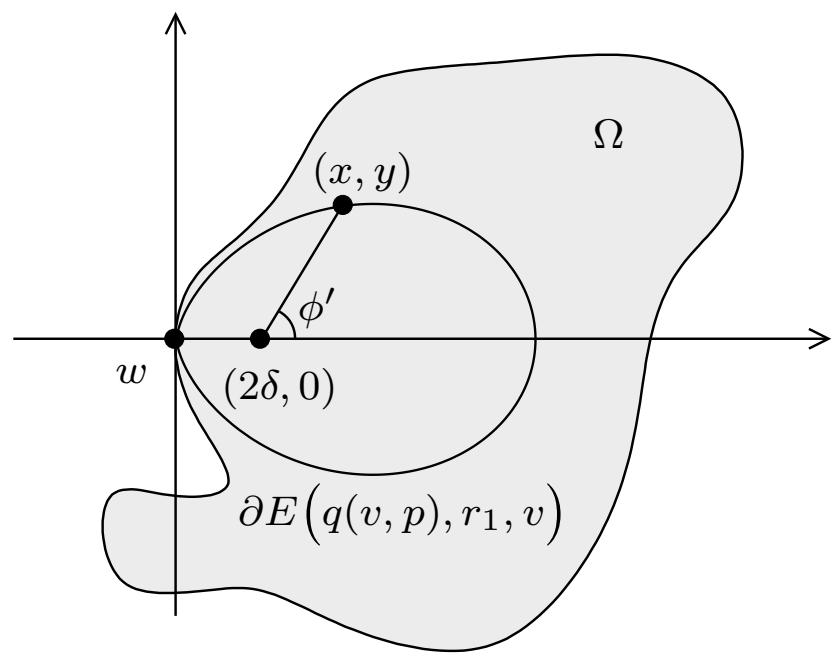

Figure 3. Geometry for the lower estimate of $\omega_{p}\left(A_{\delta}, w_{0}\right)$, part (ii) of Theorem 2.1. The function $H_{\delta}^{*}$ has a pole at $(2 \delta, 0)$, and is bounded from above by $C \delta^{q}$ on $\partial E\left(q, r_{1}, v\right)$, and by $\omega_{p}\left(A_{\delta}, w_{0}\right)$ on $\partial B\left((2 \delta, 0), \delta \tan \frac{\pi}{8 v}\right)$. In the figure $p=4$ and $a=1$.

Choice of $\delta_{0}$. Fix $\delta_{0}>0$ so small that $\delta_{0}<\frac{r_{1}}{48}$ and

$$
\frac{m_{v}}{M_{v, p}} \cdot\left(4 \delta_{0}\right)^{-q}-\frac{\pi}{2 v}\left(\frac{2}{r_{1}}\right)^{q} \geq\left(\tan \frac{\pi}{8 v}\right)^{-q}
$$

where $M_{v, p}$ and $m_{v}$ are the constants from Lemma 4.1. Note that $\delta_{0}$ can be chosen to be increasing in $p$. In the case when $p \in(1,2]$, we also assume that $\delta_{0}<r_{0}$, where $r_{0}$ is the constant from the exterior corkscrew condition (3.1). Let $\widetilde{B}_{\delta}=$ 
$\bar{B}\left((2 \delta, 0), \delta \tan \frac{\pi}{8 v}\right)$, for $\delta>0$, where $\bar{B}$ denotes the closure of $B$. We now show that, for all $\delta \in\left(0, \delta_{0}\right)$,

$$
\widetilde{B}_{\delta} \subseteq E\left(\frac{v^{2}}{2 v+1}, r_{1}, v\right) \subseteq E\left(q, r_{1}, v\right) \subseteq \Omega,
$$

where the second inclusion follows immediately since $q \geq \frac{v^{2}}{2 v+1}$, and the last inclusion holds by assumption. For the remaining inclusion, let $(r, \phi)$ be polar coordinates for $(x, y) \in \widetilde{B}_{\delta}$. Now $|\phi| \leq \frac{\pi}{8 v}$, since if $(x-2 \delta)^{2}+y^{2} \leq\left(\delta \tan \frac{\pi}{8 v}\right)^{2}$, then $x \geq \delta$, and hence also $|y| \leq x \tan \frac{\pi}{8 v}$. Consequently, $\widetilde{B}_{\delta} \subseteq E\left(\frac{v^{2}}{2 v+1}, r_{1}, v\right)$, since $\cos (v \phi) \geq \cos \left(v \cdot \frac{\pi}{8 v}\right) \geq$ $\left(\frac{r}{r_{1}}\right)^{\frac{v^{2}}{2 v+1}}$, where the last inequality holds since $r \leq 3 \delta<\frac{r_{1}}{16}$ and $\frac{v^{2}}{2 v+1} \geq \frac{1}{8}$.

Lower bound of $\omega_{p}$ on $\widetilde{B}_{\delta}$. Let $\delta \in\left(0, \delta_{0}\right)$. Aiming to find $c_{1}>0$ such that $\omega_{p}\left(A_{\delta}, \cdot\right) \geq c_{1}$ on $\widetilde{B}_{\delta}$, we first find such a lower bound near the boundary.

If $p>2$, then all points on $\partial \Omega$ are $p$-regular, see [HKM93, pages 124-125], and hence the boundary convergence in Lemma 3.1 holds. Let, for $z \in \bar{B}((0,0), \delta)$,

$$
f(z)=1-\left|\frac{z}{\delta}\right|^{\alpha}, \quad \text { where } \alpha=\alpha(p)=\frac{p-2}{p-1} \text { and } \alpha(\infty)=1 .
$$

Recall that $f$ is $p$-harmonic in $B((0,0), \delta) \backslash\{(0,0)\}$, with $f(0,0)=1$ and $f=0$ on $\partial B((0,0), \delta)$. Moreover, by Lemma 3.1 and by the comparison principle we see that $\omega_{p}\left(A_{\delta}, \cdot\right) \geq f$ whenever both functions are defined. Hence $\omega_{p}\left(A_{\delta},(\delta / 2,0)\right) \geq 1-2^{-\alpha}$.

If $p \in(1,2]$, then by assumption $\Omega$ satisfies the exterior corkscrew condition on $A_{\delta}$. This implies that $A_{\delta}$ is $p$-regular and hence we may apply Lemma 3.1 and the comparison principle. Consider the $p$-harmonic function

$$
g(x)= \begin{cases}a\left|x-x_{0}\right|^{(p-2) /(p-1)}+b, & \text { if } p \neq 2, \\ a \log \left|x-x_{0}\right|+b, & \text { if } p=2,\end{cases}
$$

for some $a, b$. Choose $a$ and $b$ such that $g$ has boundary values $g=1$ on $\partial B\left(a_{\delta / 4}(0,0)\right.$, $\delta /(4 M))$ and $g=0$ on $\partial B\left(a_{\delta / 4}(0,0), \delta / 2\right)$. Here, $M$ and $a_{\delta / 4}(\cdot)$ are given by the exterior corkscrew condition (3.1). By construction and by the comparison principle, we have $g \leq \omega_{p}\left(A_{\delta}, \cdot\right)$ whenever both functions are defined. Hence, by the Harnack inequality we conclude the existence of a constant $c$, increasing in $p$ and depending only on $M$ and $p$, such that $\omega_{p}\left(A_{\delta},(\delta, 0)\right) \geq c$.

Consequently, for all $p \in(1, \infty)$, the Harnack inequality shows that $\omega_{p}\left(A_{\delta}, \cdot\right) \geq$ $c_{1}$ on $\widetilde{B}_{\delta}$, for some constant $c_{1}>0$, depending only on $p$ and, if $p \in(1,2]$, also on $M$. Furthermore, $c_{1}$ is increasing in $p$ when $p>2$.

Upper bound of the comparison function. Let $H_{\delta}^{*}(x, y)=\frac{c_{1}}{M_{v, p}}\left(\delta \tan \frac{\pi}{8 v}\right)^{q} H_{v, p}(x-$ $2 \delta, y)$, for $(x, y) \in \mathbf{R}^{2}$ such that $(x-2 \delta, y) \in \mathcal{S}_{v}$, First note that if $(x, y) \in \partial \widetilde{B}_{\delta}$, then

$$
H_{\delta}^{*}(x, y) \leq \frac{c_{1}}{M_{v, p}}\left(\delta \tan \frac{\pi}{8 v}\right)^{q} \cdot\left(\delta \tan \frac{\pi}{8 v}\right)^{-q} \cdot M_{v, p} \leq \omega_{p}\left(A_{\delta},(x, y)\right),
$$

where the first inequality follows from property (ii) in Lemma 4.1.

Let $D$ be the set of points $(x, y) \in E\left(q, r_{1}, v\right)$ such that $(x-2 \delta, y) \in \mathcal{S}_{v}$. We now show that $H_{\delta}^{*}(x, y) \leq C_{0} \delta^{q}$ on $\partial D \backslash\{(2 \delta, 0)\}$, where

$$
C_{0}=\frac{\pi c_{1}}{2 v}\left(\frac{2}{r_{1}} \tan \frac{\pi}{8 v}\right)^{q} \text {. }
$$


Let $\left(r^{\prime}, \phi^{\prime}\right)$ be polar coordinates for $(x-2 \delta, y)$. Since $H_{v, p}(x-2 \delta, y)=\left(r^{\prime}\right)^{-q} \cdot h_{v, p}\left(\phi^{\prime}\right)$, where $h_{v, p}$ by Lemma 4.1 satisfies $h_{v, p}\left( \pm \frac{\pi}{2 v}\right)=0$ and $\left|h_{v, p}^{\prime}\right| \leq M_{v, p}$, the mean value theorem yields

$$
H_{v, p}(x-2 \delta, y) \leq M_{v, p} \cdot\left(r^{\prime}\right)^{-q}\left|\phi^{\prime} \mp \frac{\pi}{2 v}\right| .
$$

Hence, if $(x, y) \in \partial D$ and if we pick $\phi^{\prime} \in\left[-\frac{\pi}{2 v}, \frac{\pi}{2 v}\right]$, then

$$
H_{v, p}(x-2 \delta, y) \leq M_{v, p} \cdot\left(r^{\prime}\right)^{-q}\left(\frac{\pi}{2 v}-\left|\phi^{\prime}\right|\right) \leq \frac{\pi M_{v, p}}{2 v}\left(r^{\prime}\right)^{-q} \cos v \phi^{\prime} .
$$

We now show that $\cos v \phi^{\prime} \leq\left(\frac{2 r^{\prime}}{r_{1}}\right)^{q}$ on $\partial D$. If $(x-2 \delta, y) \in E\left(q, \frac{r_{1}}{2}, v\right)$, then $(2 x-4 \delta, 2 y) \in E\left(q, r_{1}, v\right)$ by definition. As the latter set is star-shaped around $(4 \delta, 0)$, we get that $(x, y)=\frac{1}{2}(2 x-4 \delta, 2 y)+\frac{1}{2}(4 \delta, 0) \in E\left(q, r_{1}, v\right)$, and hence also that $(x, y) \in D$, since $\left|\phi^{\prime}\right| \leq \frac{\pi}{2 v}$ (Figure 4). In particular, this implies that if $(x, y) \in \partial D$, then $(x-2 \delta, y) \notin E\left(q, \frac{r_{1}}{2}, v\right)$, and hence $\cos v \phi^{\prime} \leq\left(\frac{2 r^{\prime}}{r_{1}}\right)^{q}$.

By definition of $H_{\delta}^{*}$ and $C_{0}$, we then get that $H_{\delta}^{*}(x, y) \leq C_{0} \delta^{q}$ for all $(x, y) \in$ $\partial D \backslash\{(2 \delta, 0)\}$; in fact, we even have that $H_{\delta}^{*}=0$ on $\partial D \backslash \partial E\left(q, r_{1}, v\right)$.

$(0,0)$

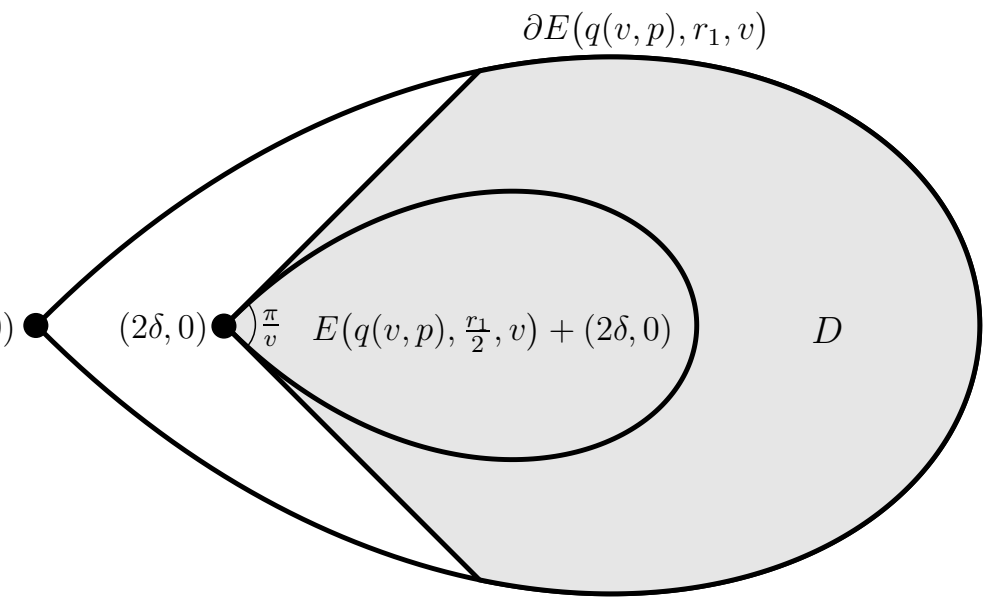

Figure 4. The shaded set $D$ is the part of $E\left(q, r_{1}, v\right)$ that lies within a sector with aperture $\frac{\pi}{v}$ and apex at $(2 \delta, 0)$. Contained in $D$ is the set $E\left(q, \frac{r_{1}}{2}, v\right)$ translated by $(2 \delta, 0)$. In the figure, $p=4$ and $v=2$.

Final comparison. Combining the last estimate with the bound of $H_{\delta}^{*}$ on $\partial \widetilde{B}_{\delta}$, the comparison principle yields that $H_{\delta}^{*} \leq \omega_{p}\left(A_{\delta}, \cdot\right)+C_{0} \delta^{q}$ on $D \backslash \widetilde{B}_{\delta}$. In particular for the point $\left(4 \delta_{0}, 0\right)$, which is in the set,

$$
\begin{aligned}
\omega_{p}\left(A_{\delta},\left(4 \delta_{0}, 0\right)\right) & \geq \frac{c_{1}}{M_{v, p}}\left(\delta \tan \frac{\pi}{8 v}\right)^{q} H_{v, p}\left(4 \delta_{0}-2 \delta, 0\right)-C_{0} \delta^{q} \\
& \geq c_{1} \delta^{q}\left(\frac{m_{v}}{M_{v, p}}\left(4 \delta_{0}\right)^{-q}-\frac{\pi}{2 v}\left(\frac{2}{r_{1}}\right)^{q}\right) \cdot\left(\tan \frac{\pi}{8 v}\right)^{q} \geq c_{1} \delta^{q},
\end{aligned}
$$

where the last inequality follows from the assumption in (4.3) on $\delta_{0}$. An application of the Harnack inequality yields $\omega_{p}\left(A_{\delta}, w_{0}\right) \geq c \omega_{p}\left(A_{\delta},\left(4 \delta_{0}, 0\right)\right)$ for some constant $c>0$ depending on $\delta_{0}$, $\operatorname{dist}\left(w_{0}, \partial \Omega\right)$ and $\left|w-w_{0}\right|$. This completes the proof of Theorem 2.1. 


\section{References}

[AKSZ07] Aikawa, H., T. Kilpeläinen, N. Shanmugalingam, and X. Zhong: Boundary Harnack principle for p-harmonic functions in smooth euclidean domains. - Potential Anal. 26, 2007, 281-301.

[AS10] Armstrong, S. N., and C. K. Smart: An easy proof of Jensen's theorem on the uniqueness of infinity harmonic functions. - Calc. Var. Partial Differential Equations 37:3-4, 2010, 381-384.

[Aro86] Aronsson, G.: Construction of singular solutions to the $p$-harmonic equation and its limit equation for $p=\infty$. - Manuscripta Math. 56:2, 1986, 135-158.

[AL88] Aronsson, G., and P. Lindqvist: On $p$-harmonic functions in the plane and their stream functions. - J. Differential Equations 74:1, 1988, 157-178.

[BL05] Bennewitz, B., and J. L. Lewis: On the dimension of $p$-harmonic measure. - Ann. Acad. Sci. Fenn. Math. 30:2, 2005, 459-505.

[Bha04] Bhattacharya, T.: On the behaviour of $\infty$-harmonic functions near isolated points. Nonlinear Anal. 58:3-4, 2004, 333-349.

[Car73] CARleson, L.: On the distortion of sets on a Jordan curve under conformal mapping. - Duke Math. J. 40, 1973, 547-559.

[CIL92] Crandall, M. G., H. Ishit, and P.-L. Lions: User's guide to viscosity solutions of second order partial differential equations. - Bull. Amer. Math. Soc. (N.S.) 27:1, 1992, $1-67$.

[GM05] Garnett, J. B., and D. E. Marshall: Harmonic measure. - New Math. Monogr. 2, Cambridge Univ. Press, Cambridge, 2005.

[HKM93] Heinonen, J., T. Kilpeläinen, and O. Martio: Nonlinear potential theory of degenerate elliptic equations. - Oxford Math. Monogr., Oxford Univ. Press, New York, 1993.

[HK97] Herron, D. A., and P. Koskela: Continuity of Sobolev functions and Dirichlet finite harmonic measures. - Potential Anal. 6:4, 1997, 347-353.

[Hir08] HiRATA, K.: Global estimates for non-symmetric Green type functions with applications to the $p$-Laplace equation. - Potential Anal. 29:3, 2008, 221-239.

[J93] JENSEN, R.: Uniqueness of Lipschitz extensions minimizing the sup-norm of the gradient. - Arch. Ration. Mech. Anal. 123, 1993, 51-74.

[Ju98] JuUtinen, P.: Minimization problems for Lipschitz functions via viscosity solutions. Ann. Acad. Sci. Fenn. Math. Diss. 115, 1998.

[KMV96] Koskela, P., J. J. Manfredi, and E. Villamor: Regularity theory and traces of $\mathcal{A}$-harmonic functions. - Trans. Amer. Math. Soc. 348:2, 1996, 755-766.

[LM95] Lindqvist, P., and J. J. Manfredi: The Harnack inequality for $\infty$-harmonic functions. - Electron. J. Differential Equations 1995:4, 1995, 1-5.

[LMW05] Llorente, J. G., J. J. Manfredi, and J.-M. Wu: p-harmonic measure is not additive on null sets. - Ann. Sc. Norm. Super. Pisa Cl. Sci. (5) 4:2, 2005, 357-373.

[LuN10] Lundström, N. L. P., and K. Nyström: The boundary Harnack inequality for solutions to equations of Aronsson type in the plane. - Ann. Acad. Sci. Fenn. Math. 36, 2011, 261-278.

[Mak85] Makarov, N. G.: On the distortion of boundary sets under conformal mappings. Proc. London Math. Soc. (3) 51:2, 1985, 369-384.

[PS08] Peres, Y., and S. Sheffield: Tug-of-war with noise: a game-theoretic view of the p-Laplacian. - Duke Math. J. 145:1, 2008, 91-120.

[PSSW09] Peres, Y., O. Schramm, S. Sheffield, and D. B. Wilson: Tug-of-war and the infinity Laplacian. - J. Amer. Math. Soc. 22:1, 2009, 167-210.

[Per89] Persson, L.: Quasi-radial solutions of the $p$-harmonic equation in the plane and their stream functions. - Licentiate Thesis 1988:14 L, Luleå Univ. of Technology, Sweden, 1989.

Received 31 May $2012 \bullet$ Accepted 27 August 2012 